\title{
PREVALENCE OF ANAPHYLACTIC REACTIONS TO APROTININ: ANALYSIS OF TWO HUNDRED FORTY-EIGHT REEXPOSURES TO APROTININ IN HEART OPERATIONS
}

\author{
W. Dietrich, MD \\ P. Späth, MD \\ A. Ebell, MD \\ J. A. Richter, MD
}

\begin{abstract}
The efficacy of aprotinin to reduce intraoperative bleeding tendency in cardiac operations has been demonstrated in several studies. Aprotinin is a polybasic polypeptide and has antigenic properties. Anaphylactic reactions to aprotinin have been described. The aim of the present study was to evaluate the prevalence of adverse reactions to reexposure to high-dose aprotinin. The clinical outcome of all patients undergoing heart operations in our institution between 1988 and 1995 with at least two exposures to aprotinin was investigated. There were 248 reexposures to aprotinin in 240 patients: 101 adult and 147 pediatric cases. The total aprotinin doses were $4.9 \times 10^{6}$ (interquartile range $2 \times 10^{6}$ ) KIU (adults) and $1.3 \times 10^{6}$ (interquartile range $1.2 \times 10^{6}$ ) $\mathrm{KIU}$ (pediatric patients). The time between the first and second aprotinin exposures was 344 (interquartile range 1039) days. Seven adverse reactions to aprotinin were found $(2.8 \%)$. The severity of the reaction ranged from mild (no intervention) to severe (longer-lasting circulatory depression despite vasopressor therapy). All patients survived the event. Patients with an interval less than 6 months since the previous exposure had a statistically higher incidence of adverse reactions than patients with a longer interval $(5 / 111$ or $4.5 \%$ vs $2 / 137$ or $1.5 \%, p<0.05)$. Two patients reacted to a test dose of 10,000 KIU aprotinin. Pretreatment with antihistaminics was done in $60 \%$ of the patients. We recommend the following procedure for reexposure with high-dose aprotinin: (1) delay of the first bolus injection of aprotinin until the surgeon is ready to begin cardiopulmonary bypass, (2) test dose of $10,000 \mathrm{KIU}$ aprotinin in all patients with aprotinin treatment, (3) $\mathrm{H}_{1} / \mathrm{H}_{2}$ blockade in known or possible reexposures, and (4) avoidance of reexposure within the first 6 months after the previous exposure to aprotinin. With these precautions a reexposure to aprotinin in patients with a high risk of bleeding is justified, because the benefits of aprotinin treatment outweigh the relative risk of a serious allergic reaction. (J Thorac Cardiovasc Surg 1997;113:194-201)
\end{abstract}

A protinin is used in cardiac operations to reduce intraoperative and postoperative bleeding tendency. ${ }^{1-5}$ It has been proved effective in this indication. ${ }^{6}$ Aprotinin, a polybasic polypeptide, is a naturally occurring inhibitor of proteolytic enzymes and consists of 58 amino acids. It has a molecular weight

From the Department of Anesthesiology, German Heart Center Munich, Munich, Germany.

Received for publication March 5, 1996; revisions requested May 8, 1996; revisions received June 6, 1996; accepted for publication July 18, 1996.

Address for reprints: Wulf Dietrich, MD, German Heart Center Munich, Department of Anesthesiology, Lazarettstraße 36, 80636 Munich, Germany.

Copyright $(1) 1997$ by Mosby-Year Book, Inc.

$0022-5223 / 97 \$ 5.00+0 \quad \mathbf{1 2} / \mathbf{1} / \mathbf{7 6 8 3 2}$ of 6500 daltons and is derived from bovine lungs. As an allogeneic protein it possesses antigenic properties and the possibility of an adverse reaction to the agent exists. Allergic reactions after reexposure to high-dose aprotinin have been described in case reports. $^{7-15}$ It has been shown that formation of immunoglobulin (Ig) $\mathrm{G}$ antibodies to aprotinin takes place in about $50 \%$ of all patients treated with aprotinin. ${ }^{16,17}$ Because aprotinin has been used on a broad scale in Europe during the past 8 years in many cardiac centers, the number of patients scheduled for a second cardiac operation after a primary exposure to aprotinin is increasing. Aprotinin is especially efficacious in cardiac reoperations ${ }^{1,18}$; however, the question remains whether the relative risk of an anaphylactic reaction might outweigh the 
benefit of this drug if it is given to previously sensitized patients.

The present study was done to evaluate the prevalence of adverse reactions after reexposure to aprotinin and to develop guidelines to reduce the risk and sequelae of such a reaction.

\section{Methods}

With institutional approval the charts of patients who underwent a cardiac reoperation between January 1988 and March 1995 were retrospectively analyzed. Adult and pediatric patients who had cardiac operations with the use of the heart-lung machine were included. Patients up to age 18 years were considered as pediatric patients. All reexposures were registered; thus cases in which patients had a third exposure to aprotinin within the study period were counted as two reexposures. Data of patients with at least two exposures to aprotinin were entered into the study and were reviewed independently by two investigators (W.D., A.E.). An allergic reaction was assumed if at least one of the following symptoms was present within 10 minutes after aprotinin administration: (1) a decrease of systolic blood pressure greater than $20 \%$ from the baseline value, (2) a change of heart rate greater than $20 \%$ from the baseline value, (3) an increase of inspiratory pressure greater than $5 \mathrm{~cm} \mathrm{H}_{2} \mathrm{O}$, and (4) a skin reaction.

Anesthesia was done with a standard intravenous technique with either fentanyl or sufentanil, midazolam, and pancuronium for muscle relaxation. A bubble oxygenator was used in almost all patients. Priming for adult patients consisted of $1400 \mathrm{ml}$ crystalloid solution. Myocardial preservation was achieved by infusion of $1000 \mathrm{ml}$ cold crystalloid cardioplegic solution (Bretschneider HTG, F. Köhler Chemie, Alsbach, Germany). Cardiopulmonary bypass (CPB) was done with moderate hypothermia of $30^{\circ}$ to $32^{\circ} \mathrm{C}$ rectal temperature and a flow rate of $2.4 \mathrm{~L} / \mathrm{min}^{-1}$ / $\mathrm{m}^{-2}$. Priming of the oxygenator for the pediatric patients was done according to the body weight with crystalloids or blood. Pediatric $\mathrm{CPB}$ was done with hypothermia of $26^{\circ}$ to $28^{\circ} \mathrm{C}$ rectal temperature and a flow rate of 1.2 to 2.4 $\mathrm{L} / \mathrm{min}^{-1} / \mathrm{m}^{-2}$ or with deep hypothermia $\left(20^{\circ} \mathrm{C}\right.$ rectal temperature) and circulatory arrest. Anticoagulation for $\mathrm{CPB}$ was done with porcine mucosa heparin, $375 \mathrm{U} / \mathrm{kg}$. Anticoagulation was controlled with the celite activated clotting time.

Aprotinin dosage was as follows. A test dose of 10,000 KIU aprotinin was given 10 minutes before the first bolus. Adult patients received an initial bolus of $2 \times 10^{6} \mathrm{KIU}$ aprotinin with the start of operation followed by a continuous infusion of $5 \times 10^{5} \mathrm{KIU} / \mathrm{hr}$. Additionally, $2 \times 10^{6}$ KIU was added to the pump prime. Pediatric patients received a bolus of aprotinin, $30,000 \mathrm{KIU} / \mathrm{kg}$, after induction of anesthesia and the same dose was added to the pump prime. The dosage regimen changed during the study period insofar as the first aprotinin dose was delayed until the surgeon was ready to cannulate the aorta and begin CPB immediately in case of a severe adverse reaction.

Reactions to aprotinin were classified as mild (no intervention), moderate (restoration of circulation within
Table I. Type of operations

\begin{tabular}{lcc}
\hline & Adults & Children \\
\hline CABG & 20 & 0 \\
Valve rereplacement & 63 & 18 \\
Conduit or shunt (with CPB) & 4 & 44 \\
HTX & 3 & 0 \\
Correction of congenital defects & 10 & 84 \\
Miscellaneous & $\underline{1}$ & $\underline{1}$ \\
$\quad$ Total & 101 & 147 \\
\hline
\end{tabular}

$C A B G$, Coronary artery bypass grafting; $H T X$, heart transplantation.

15 minutes after the event by the use of vasopressors), or severe (longer-lasting circulatory depression and instability despite vasopressor therapy). These reactions were registered regardless of surgical manipulations on the heart or the great vessels. The regimen of prophylactic antihistaminic blockade was changed during the study period: during the first years $\mathrm{H}_{1} / \mathrm{H}_{2}$ blockade (clemastine $0.03 \mathrm{mg} / \mathrm{kg}$, cimetidine $5 \mathrm{mg} / \mathrm{kg}$ ) was given at the discretion of the anesthesiologist; since January $1993 \mathrm{H}_{1} / \mathrm{H}_{2}$ blockade was given to all patients with a reexposure to aprotinin. Additionally, almost all pediatric patients were treated with dexamethasone $(1 \mathrm{mg} / \mathrm{kg})$.

Parametric data are reported as median and interquartile range (IQR). Differences in patient demographics were determined by Student's $t$ test for continuous variables and $\chi^{2}$ or Fisher's exact test for dichotomous variables with $p<0.05$ as significant (Systat 5.2 for the Macintosh computer, Systat, Evanston, Ill.).

\section{Results}

More than 6000 patients undergoing heart operations were treated with high-dose aprotinin within the study period in this institution. Among these there were 248 reexposures to aprotinin in 240 patients in 101 operations on adult patients and 147 operations on pediatric patients. The ages of the patients were 59.5 (IQR 16.2) years (adult patients) and 5.2 (2.4) years (pediatric patients), and the body weights were 67 (IQR 12.5) kg (adult patients) and 10.2 (12.5) $\mathrm{kg}$ (pediatric patients). The time between the first and the second aprotinin exposures was 344 (IQR 1039) days. The types of operations done are given in Table I. The total aprotinin doses were $4.9 \times 10^{6}\left(\mathrm{IQR} 2 \times 10^{6}\right) \mathrm{KIU}$ (adults) and $1.3 \times 10^{6}$ $\left(1.2 \times 10^{6}\right) \mathrm{KIU}$ (pediatric patients). Antihistaminic pretreatment with $\mathrm{H}_{1} / \mathrm{H}_{2}$ blockers was done in 141 patients, treatment with corticosteroids in $140 \mathrm{pa}$ tients, and combined treatment with both these agents in 83 patients.

Seven adverse reactions to aprotinin were found (Tables II and III), of which one was unclear and classified as a "doubtful" reaction to aprotinin (patient 1). The course of these patients is described in the following section. Two patients reacted to the 
Table II. Distribution and severity of adverse reactions to aprotinin

\begin{tabular}{lcccccc}
\hline & & \multicolumn{5}{c}{ Reactions } \\
\cline { 2 - 7 } & $n$ & Doubtful & Mild & Moderate & Severe & Total \\
\hline Adult & 101 & 1 & 2 & & 1 & 4 \\
Pediatric & 147 & & & 1 & 2 & 3 \\
$\quad$ Total & 248 & 1 & 1 & 1 & 3 & 7 \\
\hline
\end{tabular}

Explanation of severity score is given in the text.

test dose of 10,000 KIU aprotinin ( 1 mild, 1 severe; patients 6 and 7) and received no additional aprotinin. In 137 patients the time to the previous aprotinin exposure was longer than 200 days. Two patients in this group reacted to aprotinin (1.5\%). On the other hand, $5(4.5 \%)$ of 111 patients with an interval less than 200 days reacted to aprotinin $(p<$ 0.05 ) (Fig. 1). All patients with reactions survived the event; one of these patients died of multiorgan failure on postoperative day 6 as a result of the underlying disease. Mortality (30 days) of all patients was $4.8 \%$.

\section{Case reports}

Patient 1. This 71-year-old woman had coronary artery disease and aortic regurgitation. She had had a previous aortic and mitral valve replacement and triple coronary artery bypass grafting 193 days before undergoing the operation in which the reaction to aprotinin occurred. At this most recent operation she had stable angina caused by an occluded vein graft to the left anterior descending artery and paravalvular leakage around the prosthetic aortic valve. During the first operation she had been treated with $6 \times 10^{6} \mathrm{KIU}$ aprotinin. At the second operation anesthesia was induced with fentanyl and flunitrazepam. The patient received $\mathrm{H}_{1} / \mathrm{H}_{2}$ blockade (clemastine $0.03 \mathrm{mg} / \mathrm{kg}$, cimetidine $5 \mathrm{mg} / \mathrm{kg}$ ) after induction of anesthesia. Ten minutes later and before skin incision aprotinin infusion was started. After $5 \times 10^{5} \mathrm{KIU}$ aprotinin was administered the blood pressure dropped from $140 / 40$ to $100 / 40 \mathrm{~mm}$ $\mathrm{Hg}$ and the aprotinin infusion was stopped. Blood pressure returned to normal with $15 \mu \mathrm{g}$ norepinephrine administered intravenously within 5 minutes. At this time it was unclear whether the hemodynamic changes were caused by aprotinin. Therefore the aprotinin infusion was started again. Systolic blood pressure varied between 140 and $120 \mathrm{~mm} \mathrm{Hg}$. Another bolus of $5 \times 10^{5} \mathrm{KIU}$ aprotinin coincided with surgical manipulation of the heart and ventricular fibrillation occurred. After electric defibrillation the hemodynamic condition was stable, but aprotinin administration was stopped and tranexamic acid in a total dose of $4 \mathrm{gm}$ was given instead. During CPB the aortic valve was reinforced with sutures and the left anterior descending artery was supplied by the left thoracic artery. After termination of $\mathrm{CPB}$ increased bleeding tendency was evident, but the patient's condition was hemodynamically stable with a $5 \mu \mathrm{g} / \mathrm{kg}^{-1} / \mathrm{min}^{-1}$ dopamine infusion. The postoperative course was complicated by increased chest tube drainage, which necessitated reexploration for surgical hemostasis 6 hours after operation. The patient recovered uneventfully. Because of the coincidence of hemodynamic instability with surgical manipulation and the uncertain relation to aprotinin infusion this case was classified as a doubtful reaction to aprotinin.

Patient 2. This 82-year-old female patient had mitral insufficiency. Operation for mitral valve repair during which she had received $5.5 \times 10^{6} \mathrm{KIU}$ aprotinin had been done 127 days before the most recent operation. Because mitral regurgitation resumed shortly after the first operation a second operation was mandatory. Before operation the patient had dyspnea at rest and pulmonary congestion. After induction of anesthesia with sufentanil and midazolam she received $\mathrm{H}_{1} / \mathrm{H}_{2}$ blockade. During skin incision aprotinin infusion was started. After $5 \times 10^{5} \mathrm{KIU}$ aprotinin was administered the systolic blood pressure dropped from 90 to $50 \mathrm{~mm}$ $\mathrm{Hg}$ but was restored within 5 minutes with $50 \mu \mathrm{g}$ norepinephrine administered intravenously. Aprotinin infusion was stopped. Corticosteroids $(500 \mathrm{mg}$ methylprednisolone) were given. Because of difficult preparation it took 90 minutes until the start of CPB. During this time the systolic blood pressure was stable between 90 and $70 \mathrm{~mm} \mathrm{Hg}$ with inotropic support of $5 \mu \mathrm{g} / \mathrm{kg}^{-1} / \mathrm{min}^{-1}$ dopamine. During a CPB time of 59 minutes mitral regurgitation was corrected. After termination of CPB the patient's circulatory condition was unstable despite inotropic support. After operation, prolonged artificial ventilation and hemodialysis were necessary. The patient died 6 days after operation of multiorgan failure. The contribution of the reaction to aprotinin to the fatal outcome remains unclear. This case was classified as severe reaction to aprotinin.

Patient 3. This 5-year-old female patient (body weight $18 \mathrm{~kg}$ ) had a double-outlet right ventricle with hypoplastic left ventricle and a common atrioventricular valve. Five years before the most recent operation she had undergone resection of the right outflow tract without aprotinin therapy. Twenty- 


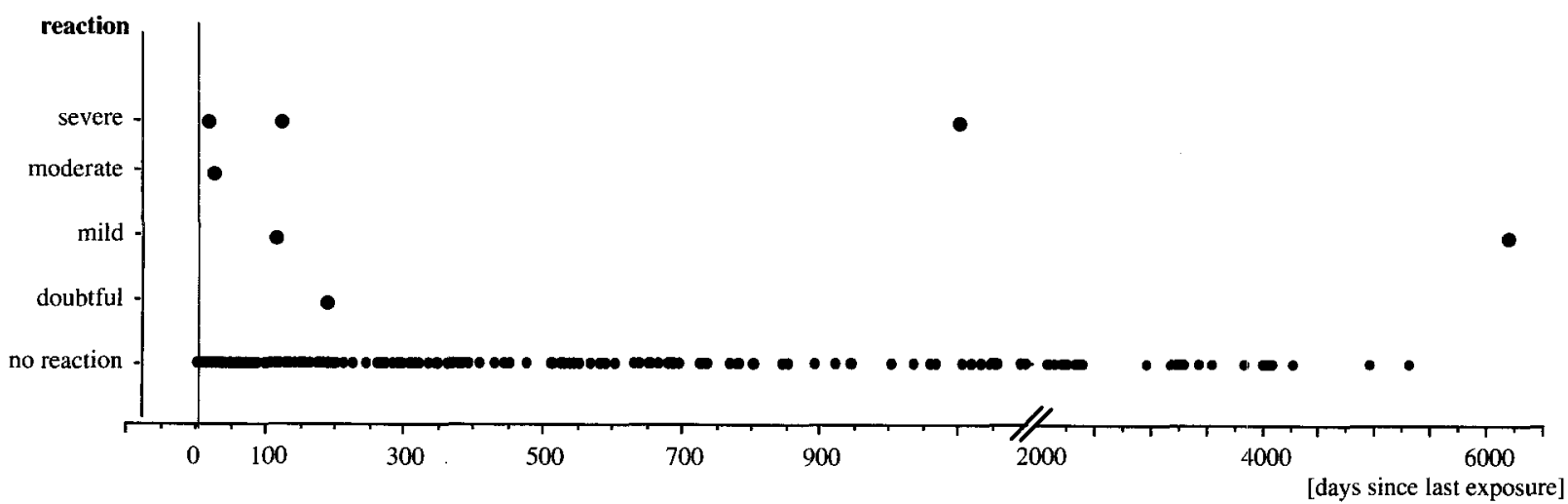

Fig. 1. Graph shows interval between most recent and prior aprotinin treatments. Cases with reactions are marked with large dots. Five of seven reactions occurred within 200 days after previous exposure. See text for explanation of severity score.

Table III. Data of patients with adverse reactions

\begin{tabular}{|c|c|c|c|c|c|c|c|c|}
\hline $\begin{array}{l}\text { Pt. } \\
\text { No. }\end{array}$ & $\begin{array}{l}\text { Age } \\
(y r)\end{array}$ & Diagnosis & Operation & $\begin{array}{l}\text { Time to most } \\
\text { recent exposure }\end{array}$ & $\begin{array}{c}\text { Test } \\
\text { doses }\end{array}$ & $\begin{array}{c}H_{1} / H_{2} \\
\text { blockers* }\end{array}$ & Corticosteroids* & Reaction \\
\hline 1 & 71 & Aortic regurgitation & Valve replacement & 193 days & No & Yes & No & Doubtful \\
\hline 2 & 82 & Mitral insufficiency & Valve replacement & 127 days & No & Yes & No & Severe \\
\hline 3 & 5 & DORV & Cavopulmonary anastomosis & 29 days & No & Yes & Yes & Moderate \\
\hline 4 & 13 & PA & RV-PA conduit & 1106 days & Yes & No & No & Severe \\
\hline 5 & 20 & TOF & RV-PA conduit & $19 \mathrm{yr}$ & Yes & No & No & Mild \\
\hline 6 & 66 & Mitral insufficiency & Valve replacement & 120 days & Yest & Yes & No & Mild \\
\hline 7 & 7 & PA & Aortopulmonary shunt & 22 days & Yest & Yes & No & Severe \\
\hline
\end{tabular}

$\overline{D O R V}$, Double-outlet right ventricle; $P A$, pulmonary atresia; $R V-P A$, right ventricle-pulmonary artery; $T O F$, tetralogy of Fallot.

${ }^{*}$ Given before the test dose of aprotinin.

$\uparrow$ Reaction to test dose.

nine days before the most recent operation a cavopulmonary anastomosis was done. During that operation she was treated with $9 \times 10^{5}$ KIU aprotinin. Because the flow to the lungs was not satisfactory a revision of the cavopulmonary anastomosis was necessary. After induction of anesthesia with fentanyl and flunitrazepam $250 \mathrm{mg}$ methylprednisolone and $\mathrm{H}_{1} / \mathrm{H}_{2}$ blockers were given. During skin incision $40,000 \mathrm{KIU}$ aprotinin was infused. Five minutes later the systolic blood pressure dropped from 120 to $80 \mathrm{~mm} \mathrm{Hg}$ and the heart rate increased from 100 to $150 \mathrm{~min}^{-1}$. There was no visible skin reaction. Aprotinin infusion was stopped. Blood pressure recovered within 10 minutes with $5 \mu \mathrm{g}$ epinephrine administered intravenously. CPB was begun 70 minutes later. No more aprotinin was given and the further course of operation was uneventful. This case was classified as moderate reaction to aprotinin.

Patient 4. This 13-year-old female patient had pulmonary atresia. Three years before the operation in which reaction to aprotinin occurred a right ventricle-pulmonary artery conduit had been placed. During that operation she had received $1.4 \times 10^{6} \mathrm{KIU}$ aprotinin. Most recently the patient underwent replacement of the calcified conduit. After induction of anesthesia with fentanyl and flunitrazepam $6 \mathrm{mg}$ dexamethasone was given. Before skin incision the patient received 50,000 KIU aprotinin as a test dose without adverse reaction. Ten minutes later infusion of $1.3 \times 10^{6} \mathrm{KIU}$ aprotinin over 10 minutes was begun. Five minutes later the systolic blood pressure dropped from 110 to 60 $\mathrm{mm}$ Hg. Despite massive inotropic support the mean arterial pressure remained between 30 and 40 $\mathrm{mm}$ Hg. Operation was started immediately and commencement of CPB was possible after $45 \mathrm{~min}$ utes of preparation. The patient was cooled to a core temperature of $16.9^{\circ} \mathrm{C}$ and the conduit replacement was done with the use of deep hypothermic circulatory arrest lasting 57 minutes. Total CPB time was 153 minutes. After termination of CPB 
circulation recovered slowly with inotropic support of $7.5 \mu \mathrm{g} / \mathrm{kg}^{-1} / \mathrm{min}^{-1}$ dopamine. The postoperative course was uneventful. This case was classified as severe reaction to aprotinin.

Patient 5. This female patient was operated on 19 years previously for correction of tetralogy of Fallot. There was no information available about aprotinin therapy during that operation; however, it was usual at that time to treat patients in the postoperative period with small doses $\left(1\right.$ to $\left.5 \times 10^{5} \mathrm{KIU}\right)$ of aprotinin. The most recent operation was scheduled for closure of a residual ventricular septal defect and for placement of a right ventricle-pulmonary artery conduit. Anesthesia was induced with fentanyl and flunitrazepam, and the patient received a test dose of $10,000 \mathrm{KIU}$ aprotinin, which was tolerated without adverse reactions. After sternotomy an infusion of aprotinin was started. When $1.5 \times 10^{6} \mathrm{KIU}$ aprotinin had been infused a slight drop in blood pressure was noted. Shortly after, flush and wheals on the forehead were seen. Peripheral arterial oxygen saturation dropped from $95 \%$ to $85 \%$. Systolic blood pressure decreased from 100 to $80 \mathrm{~mm} \mathrm{Hg}$ but recovered spontaneously within a few minutes. Corticosteroids $(250 \mathrm{mg})$ and $\mathrm{H}_{1} / \mathrm{H}_{2}$ blockers were given at this time. To reduce a suspected right-to-left shunt norepinephrine $(10 \mu \mathrm{g})$ was given 30 minutes after the event and oxygen saturation recovered to 93\%. Aprotinin infusion was stopped. The oxygenator had already been primed with $2 \times 10^{6} \mathrm{KIU}$ aprotinin. Thus the oxygenator had to be replaced and filled with an aprotinin-free prime. Circulation remained stable and the operation was done uneventfully without further sequelae. This case was classified as mild reaction to aprotinin.

Patient 6. This 66-year-old patient had mitral regurgitation. He had had a previous mitral valve replacement 120 days before undergoing the operation in which a reaction to aprotinin occurred. The most recent operation was done for repair of a leakage on the mitral valve and aortic valve replacement to correct aortic valve insufficiency. During the previous uneventful operation he had received $6 \times$ $10^{6} \mathrm{KIU}$ aprotinin. In the most recent operation, after standard induction of anesthesia with sufentanil and midazolam $\mathrm{H}_{1} / \mathrm{H}_{2}$ blockade was done. During skin incision a test dose of $10,000 \mathrm{KIU}$ aprotinin was given. Shortly after, a skin flush developed, but the patient's condition remained hemodynamically stable. No further aprotinin was given and the operation was continued routinely. Perfusion pressure during CPB was remarkably low. This case was classified as mild reaction to aprotinin.
Patient 7. This 7-year-old boy with a body weight of $19 \mathrm{~kg}$ had pulmonary atresia and ventricular septal defect. Other complex lesions rendered primary correction of the defect impossible. In his first year of life an aortopulmonary shunt was placed. The shunt was renewed 4 years before the most recent operation. During that operation the patient received a total of $80,000 \mathrm{KIU}$ aprotinin. In a third operation 3 weeks before the most recent operation a replacement of the aortopulmonary shunt was done. During this operation $1.5 \times 10^{6} \mathrm{KIU}$ aprotinin was given after injection of $\mathrm{H}_{1} / \mathrm{H}_{2}$ blockade and $8 \mathrm{mg}$ dexamethasone. The patient tolerated this reexposure to aprotinin without sequelae. Oxygen saturation remained poor after operation. Therefore a new shunt revision was scheduled 3 weeks later. $\mathrm{H}_{1} / \mathrm{H}_{2}$ blockade was given before this most recent operation. Thirty minutes after the start of the operation a test dose of $10,000 \mathrm{KIU}$ aprotinin was injected. Immediately the systolic blood pressure dropped from 100 to $70 \mathrm{~mm} \mathrm{Hg}$. The heart rate increased from 100 to $180 \mathrm{~min}^{-1}$. Venous cannulation for $\mathrm{CPB}$ was difficult because of severe bleeding from the site of cannulation. Despite $50 \mu \mathrm{g}$ epinephrine the blood pressure remained $80 / 40 \mathrm{~mm} \mathrm{Hg}$. Blood loss at this time was severe. $\mathrm{CPB}$ was begun 20 minutes after aprotinin injection. After termination of CPB inotropic support with norepinephrine and dopamine was required. The postoperative period was uneventful. This incident was classified as severe reaction to aprotinin.

\section{Discussion}

The present investigation is a retrospective study. However, our data collection is extensive and complete since 1988. Additionally, because we are aware of the possibility of an adverse reaction to aproti$\operatorname{nin}^{19}$ the reports about these reactions are carefully collected. Accordingly, the data are accurate and this report completely describes our experience with aprotinin reexposures during the past 7 years. We use aprotinin only in patients undergoing CPB. Because we have noted beneficial effects of aprotinin in pediatric heart operations, ${ }^{20}$ the percentage of pediatric patients in our study is high. The prevalence of adverse drug reactions to aprotinin reexposure was $7(2.8 \%)$ of 248 . This number differs from those of other reports: Schulze and associates $^{12}$ reported a prevalence of $5.8 \%$ for allergic reactions after aprotinin reexposure, Diefenbach and associates ${ }^{10}$ a frequency of $6 \%$, and Ceriana and colleagues $^{8} 1$ patient in 200 reexposures. This difference may be explained by incomplete data col- 
lection either because of missed reexposures without sequelae or overlooked mild reactions to aprotinin. Of note, in our patients there was a wide variation in the severity of the symptoms of the adverse events and it was not always unequivocally possible to relate hemodynamic instability to a reaction to aprotinin.

As a protein aprotinin has antigenic properties. ${ }^{21}$ Aprotinin has similarities to protamine: both have nearly identical molecular weights, are isolated from animal sources, and have been implicated in producing anaphylactic reactions. ${ }^{22}$ Anaphylactic reactions have been described since the clinical use of this drug. ${ }^{23}$ Weipert and colleagues ${ }^{17}$ demonstrated that in $50 \%$ of patients with high-dose aprotinin treatment IgG antibodies to aprotinin develop within 3 months after operation. After 48 months $50 \%$ of all patients still showed measurable levels of IgG against aprotinin. These results have been confirmed by others. ${ }^{16}$ The formation of IgE antibodies against aprotinin, which are responsible for the immediate-type allergic reaction, has also been described. ${ }^{9,10}$ If the reactions to aprotinin are antibody mediated, the assessment of drug-specific antibodies before treatment may identify patients at risk. However, a clinically useful, reliable, and predictive test system does not yet exist.

Skin testing, either by prick or intradermal test, is an established method to identify patients hypersensitive to histamine-releasing drugs such as penicil$\operatorname{lin}^{24}$ and streptokinase. ${ }^{25}$ However, in our experience, results of prick tests with pure, undiluted aprotinin before operation were not encouraging as they were in other centers. ${ }^{16}$ In our hands, results were false-negative in three patients with intraoperative reaction to aprotinin; none of our prick test results was positive so far. On the other hand, Dewachter and coworkers ${ }^{9}$ reported a positive intradermal reaction 6 weeks after an adverse reaction to aprotinin.

The use of prophylactic antianaphylactic medication and the timing of aprotinin administration changed over the years in our institution: $\mathrm{H}_{1} / \mathrm{H}_{2}$ blockade (clemastine $0.03 \mathrm{mg} / \mathrm{kg}$, cimetidine $5 \mathrm{mg} /$ $\mathrm{kg}$ ) is now used in all patients with a reexposure to aprotinin. The rationale for this treatment is based on the fact that histamine mediates anaphylactic reactions. ${ }^{26}$ Lorenz and colleagues ${ }^{27}$ demonstrated that the prophylactic use of $\mathrm{H}_{1} / \mathrm{H}_{2}$ antagonists reduced histamine-related disturbances in patients treated with gelatine infusion from $26 \%$ in the control group to $2 \%$ in the group treated with $\mathrm{H}_{1} / \mathrm{H}_{2}$ antagonists. The potential impact of $\mathrm{H}_{1} / \mathrm{H}_{2}$ blockade has also been demonstrated in anaphylactoid reactions to radiographic contrast media. ${ }^{28}$ Six of our seven patients with adverse reactions received $\mathrm{H}_{1} / \mathrm{H}_{2}$ blockers. We can only speculate whether this treatment attenuated or blunted the reaction to aprotinin, but it is remarkable that the prevalence and severity of the events in our study were less pronounced than those described in other case reports. ${ }^{8,10,12}$ Aprotinin, like other polypeptides, has been implicated as a histamine releaser from mast cells. ${ }^{21}$ Thus it seems to be prudent to reduce histamine liberation before the treatment. ${ }^{27,29}$ In cases of aprotinin reexposure, $\mathrm{H}_{1} / \mathrm{H}_{2}$ blockade, though not yet proved in a controlled study to be effective, is recommended. The role of pharmacologic prophylaxis with corticosteroids is not yet established.

Two of our patients reacted after the test dose of $10,000 \mathrm{KIU}$ aprotinin. One of these reactions was mild (patient 6) and the other one was severe but coincided with severe surgical bleeding (patient 7). We strongly recommend an aprotinin test dose be administered before the loading dose. It is advisable to prime the heart-lung machine with aprotinin only after the test dose is given because otherwise in case of a reaction to the test dose an exchange of the oxygenator will be mandatory (patient 5). A test dose should also be given before CPB if only the oxygenator is primed with aprotinin ("low-dose regimen"), because an anaphylactic reaction also may occur during CPB.

We delayed the test and the loading doses of aprotinin until the surgeon was ready to immediately begin $\mathrm{CPB}$. Hemostatic activation during the time before $\mathrm{CPB}$ is not comparable to the activation that occurs during $\mathrm{CPB},{ }^{30}$ and the influence of aprotinin in this period, if any, is minimal. On the other hand, CPB may be lifesaving in face of a circulatory breakdown caused by anaphylaxis. Especially in repeat operations the interval between skin incision and the start of CPB can be prolonged because of difficult preparation. Patients 2 and 4 had longer-lasting circulatory suppression after the adverse event because immediate commencement of $\mathrm{CPB}$ was not feasible. Some of the severe reactions to aprotinin reported in the literature occurred when the drug was given before skin incision or without a test dose. $7,10,12$

A remarkable time-dependent risk of anaphylactic reactions exists. The prevalence of reactions was higher in patients with a reexposure interval less than 200 days $(4.5 \%$ vs $1.5 \%$ in patients with a longer drug-free interval, $p<0.05$ ). In this period 
aprotinin reexposure should be avoided and, if it seems to be unavoidable, must be done with all precautions. The majority of case reports of adverse events describe a short interval of 35 days to 2 months between the first and second aprotinin exposures. Patient 7 provides an example of this time dependency: his first reexposure after 4 years was uneventful, but during the second reexposure 21 days later even the test dose caused severe hemodynamic instability.

It is unknown yet whether a dose dependency of the adverse reaction exists. However, it is conceivable that every contact with the drug regardless of the dose is antigenic. Of note, some fibrin glue preparations used outside North America contain aprotinin, which may also cause sensitization to aprotinin, ${ }^{31}$ whereas the preparations of fibrin glue within North America are being formulated without either aprotinin or $\epsilon$-aminocaproic acid. ${ }^{32}$

In conclusion, the prevalence of adverse reactions to aprotinin during reexposures in the present study was $7(2.8 \%)$ of 248 . A reliable test system to identify patients at risk does not yet exist. We recommend the following procedure to reduce the risk and severity of adverse reactions to aprotinin: (1) delay of first bolus injection of aprotinin until the surgeon is ready to begin CPB, (2) test dose of $10,000 \mathrm{KJU}$ aprotinin in all patients with aprotinin treatment (not restricted to reexposures), (3) $\mathrm{H}_{1} / \mathrm{H}_{2}$ blockade in known or possible reexposures, and (4) avoidance of reexposure within the first 6 months after the last exposure. With these precautions a reexposure to aprotinin in patients with a high risk of bleeding is justified, and the benefits of aprotinin treatment outweigh the relative risk of a serious allergic reaction.

\section{Addendum}

Since the end of the study period in March 1995, an additional 134 patients underwent aprotinin reexposure in our institution (45 adults and 89 pediatric cardiac patients). One severe adverse event occurred (19 days after sensitization). Thus until October 1996 we have had 382 reexposures with eight adverse reactions to aprotinin (i.e., an incidence of $2.1 \%$ ).

\section{REFERENCES}

1. Cosgrove DM, Heric B, Lytle BW, et al. Aprotinin therapy for reoperative myocardial revascularization: a placebo-controlled study. Ann Thorac Surg 1992;54:1031-8.

2. Bidstrup BP, Royston D, Sapsfort RN, Taylor KM. Reduction in blood loss and blood use after cardiopulmonary bypass with high dose aprotinin (Trasylol). J Thorac Cardiovase Surg 1989;97:364-72.
3. Dietrich W, Dilthey G, Spannagl M, Jochum M, Braun SL, Richter JA. Influence of high-dose aprotinin on anticoagulation, heparin requirement, and celite- and kaolin-activated clotting time in heparin-pretreated patients undergoing open-heart surgery: a double-blind, placebo-controlled study. Anesthesiology 1995;83:679-89.

4. Lemmer JH, Stanford W, Bonney SL, et al. Aprotinin for coronary bypass operations: efficacy, safety, and influence on early saphenous vein graft patency-a multicenter, randomized, double-blind, placebo-controlled study. J Thorac Cardiovase Surg 1994;107:543-53.

5. Murkin JM, Lux J, Shannon NA, et al. Aprotinin significantly decreases bleeding and transfusion requirements in patients receiving aspirin and undergoing cardiac operations. J Thorac Cardiovasc Surg 1994;107:554-61.

6. Dietrich W, Barankay A, Hahnel C, Richter JA. High-dose aprotinin in cardiac surgery: three years' experience in 1,784 patients. J Cardiothorac Vasc Anesth 1992;6:324-7.

7. Böhrer H, Bach A, Fleischer F, Lang J. Adverse haemodynamic effects of high-dose aprotinin in a paediatric cardiac surgical patient. Anaesthesia 1990;45:853-4.

8. Ceriana P, Maurelli M, Locatelli A, Bianchi T, Maccario R, De Amici M. Anaphylactic reaction to aprotinin [Letter]. J Cardiothorac Vasc Anesth 1995;9:477-84.

9. Dewachter P, Mouton C, Masson C, Gueant JL, Haberer JP. Anaphylactic reaction to aprotinin during cardiac surgery [Letter]. Anaesthesia 1993;48:1110-1.

10. Diefenbach C, Abel M, Limpers B, et al. Fatal anaphylactic shock after aprotinin reexposure in cardiac surgery. Anesth Analg 1995;80:830-1.

11. Freeman JG, Turner GA, Venables CW, Latner AL. Serial use of aprotinin and incidence of allergic reactions. Curr Med Res Opin 1983;8:559-61.

12. Schulze K, Graeter T, Schaps D, Hausen B. Severe anaphylactic shock due to repeated application of aprotinin in patients following intrathoracic aortic replacement. Eur J Cardiothorac Surg 1993; 7:495-6.

13. Schuler TM, Frosch PJ, Arza D, Wahl R. Allergie vom Soforttyp: anaphylaktische Reaktion auf Aprotinin. München Med Wochenschr 1987;129:816-7.

14. Yanagihara $Y$, Shida T. Immunological studies on patients who received aprotinin therapy. Jpn J Allergol 1985;34:899904.

15. Wuthrich B, Schmid P, Schmid ER, Tornic M, Johansson SG. $\mathrm{IgE}$-mediated anaphylactic reaction to aprotinin during anaesthesia [Letter]. Lancet 1992;340:173-4.

16. Pfannschmidt J, Steeg D, Jugert F. Routine intraoperative application of high-dose aprotinin in open heart surgery in adults: antibody formation after first exposure. Curr Med Res Opin 1995;13:282-4.

17. Weipert J, Jochum M, Dietrich W, Sebening F. Long time follow-up of aprotinin specific antibodies after cardiac surgery (abstract). Thorac Cardiovasc Surg 1995;43:132.

18. Levy JH, Pifarre R, Schaff HV, et al. A multicenter, doubleblind, placebo-controlled trial of aprotinin for reducing blood loss and the requirement for donor-blood transfusion in patients undergoing repeat coronary artery bypass grafting. Circulation 1995;92:2236-44.

19. Dietrich W, Barankay A, Dilthey G, et al. Reduction of homologous blood requirement in cardiac surgery by intraoperative aprotinin application: clinical experience in 152 
cardiac surgical patients. Thorac Cardiovasc Surg 1989;37: 92-8.

20. Dietrich W, Mossinger H, Spannagl M, et al. Hemostatic activation during cardiopulmonary bypass with different aprotinin dosages in pediatric patients having cardiac operations. J Thorac Cardiovasc Surg 1993;105:712-20.

21. Levy JH. Antibody formation after drug administration during cardiac surgery: parameters for aprotinin use. J Heart Lung Transplant 1993;12:S26-33.

22. Levy H, Schwieger I, Zaidan J, Faraj B, Weintraub W. Evaluation of patients at risk for protamine reactions. J Thorac Cardiovasc Surg 1989;98:200-4.

23. Proud G, Chamberlain J. Anaphylactic reaction to aprotinin. Lancet 1976;II:48-9.

24. Gadde J, Spence M, Wheeler B, Adkinson NF Jr. Clinical experience with penicillin skin testing in a large inner-city STD clinic. JAMA 1993;270:2456-63.

25. Dykewicz MS, McGrath KG, Davison R, Kaplan KJ, Patterson R. Identification of patients at risk for anaphylaxis due to streptokinase. Arch Intern Med 1986;146:305-7.

26. Lorenz W, Ennis M, Doenicke A, Dick W. Perioperative use of histamine antagonists. J Clin Anesth 1990;2:345-60.
27. Lorenz W, Duda D, Dick W, et al. Incidence and clinical importance of perioperative histamine release: randomized study of volume loading and antihistamines after induction of anesthesia. Lancet 1994;343:933-40.

28. Ring J, Rothenberger K, Clauss W. Prevention of anaphylactoid reactions after radiographic contrast media infusion by combined histamine H1- and H2-receptor antagonists: results of a prospective controlled trial. Int Arch Allergy Immunol 1985;78:9-14.

29. Silverman HJ, Taylor WR, Smith PL, et al. Effects of antihistamines on the cardiopulmonary changes due to canine anaphylaxis. J Appl Physiol 1988;64:210-7.

30. Dietrich W, Spannagl M, Jochum $M$, et al. Influence of high-dose aprotinin treatment on blood loss and coagulation pattern in patients undergoing myocardial revascularization. Anesthesiology 1990;73:1119-26.

31. Kon NF, Masumo H, Nakajima S, Tozawa R, Kimura M, Maeda S. Anaphylactic reaction to aprotinin following topical use of biological tissue sealant. Masui 1994;43:1606-10.

32. Alving BM, Weinstein MJ, Finlayson JS, Menitove JE, Fratantoni JC. Fibrin sealant: summary of a conference on characteristics and clinical uses. Transfusion 1995;35:783-90. 